Sharif University of Technology
Scientia Iranica
SCIENTIA $\quad \begin{gathered}\text { Transactions D: Computer Science ES Engineering and Electrical Engineering } \\ \text { w }\end{gathered}$

\title{
Amplitude reconstruction of clipped OFDM by using DFT-based least squares
}

\author{
A. Ahmadi ${ }^{a, *}$ and S. Talebi $i^{a, b}$ \\ a. Department of Electrical Engineering, Shahid Bahonar University of Kerman, Kerman, P.O. Box 76169-133, Iran. \\ b. Advanced Communications Research Institute, Sharif University of Technology, Tehran, Iran. \\ Received 23 July 2014; received in revised form 27 August 2015; accepted 26 January 2016
}

\section{KEYWORDS}

OFDM;

PAPR;

Clipping;

DFT-based least

squares;

Fast amplitude

reconstruction.

\begin{abstract}
OFDM is an effective multicarrier transmission technique with one primary disadvantage; it suffers from high Peak-to-Average Power Ratio (PAPR). Although clipping and filtering is a simple and effective method for PAPR reduction, it makes in-band and out-of-band noise, which degrades the bit error rate performance and spectral efficiency. Publications on this subject show that clipped samples could be reconstructed at the receiver by using oversampled signal and bandwidth expansion. By building on published literature, this paper aims to achieve a low-complexity method. The proposed method has complexity order of $O\left(L^{2}\right)$ to solve linear system, where $L$ indicates the number of clipped samples. Simulation results confirm that our proposed method leads to both a better biterror rate performance and a lower complexity than similar methods. These results also show that our method offers adequate performance, especially at low clipping ratios.

(C) 2017 Sharif University of Technology. All rights reserved.
\end{abstract}

\section{Introduction}

Orthogonal Frequency-Division Multiplexing (OFDM) is an effective multi-carrier transmission technique with attractive features such as high spectral efficiency and robustness of multipath fading channels; however, the Peak-to-Average Power Ratio (PAPR) is a major drawback of the OFDM technique. A survey of published papers details recent advances in this field, including several attempts to overcome the PAPR shortcoming such as clipping and filtering [1,2], Partial Transmit Sequence (PTS) [3,4], Selected Mapping (SLM) [5], active constellation extension [6], companding [7], etc. Among these methods, clipping and filtering seems to be one of the simplest and most effective solutions, especially when the number of OFDM subcarriers is large.

\footnotetext{
*. Corresponding author. Tel.: +983431322505; Fax: +98 34-33257500

E-mail addresses: adel.ahmadi@ieee.org (A. Ahmadi); siamak.talebi@uk.ac.ir (S.Talebi)
}

Clipping can be performed at Nyquist rate or higher. If signal is clipped at Nyquist rate, peak regrowth occurs after Digital-to-Analog (D/A) conversion. On the other hand, though oversampled signal clipping reduces the peak regrowth after D/A conversion, it causes out-of-band radiation. Further, clipping procedure generates in-band and out-of-band noise. The in-band noise degrades Bit Error Rate (BER) performance and the out-of-band noise reduces spectral efficiency. In order to save spectral efficiency, out-of-band noise is filtered but the filtering operation produces in-band noise and another peak regrowth. To reduce overall peak regrowth, clipping and filtering operation can be repeated until a desired PAPR is attained. In [8], convex optimizing techniques are used in order to design optimized filters for iterative clipping and filtering procedure. Optimized filters reduce inband and out-of-band noise resulting in lower out-ofband radiation and less BER degradations compared to traditional clipping and filtering techniques. However, in this approach, computational complexity of designing optimized filters is high. 
On the other hand, there are techniques which reconstruct the clipped samples at the receiver and here is an overview of some of them. In [9], a DecisionAided Reconstruction (DAR) method is introduced in which authors perform clipping operation in baseband at Nyquist rate. At the receiver, clipping noise is mitigated iteratively by initially making decisions in the frequency domain and then converting them back to the time domain. Based on DAR, the Iterative Amplitude Reconstruction (IAR) method is proposed by Kwon et al. [10,11], which improves the DAR method and yields better BER performance. The DAR method reconstructs both amplitudes and phases of clipped samples while the IAR only reconstructs amplitudes. The IAR method uses phases of clipped samples because clipping procedure only reduces amplitudes of samples and leaves phases unaffected. Thus, the IAR method offers a better BER performance than its DAR counterpart. In [12], an iterative scheme is presented which estimates and removes the clipping noise in the frequency domain. This scheme estimates the clipping noise and cancels it at receiver.

On the other side, the lost samples of an oversampled signal can be reconstructed by building some equations based on valid samples [13-16]. In these methods, if the out-of-band components of the signal are removed completely, the reconstruction procedure fails $[15,16]$. On the basis of [14-16], a clipping noise cancellation model using oversampled signal is unveiled in [17]. In this study, first, the oversampled signal is clipped; then, some out-of-band components of the clipped signal are saved during out-of-band filtering procedure. At the receiver, the clipped samples of oversampled signal, considered as lost samples, are reconstructed by employing the least square method. The reconstruction using this method is quite robust against additive channel noise, but the penalty is high complexity.

A hybrid method was proposed in [18] that used least square method [17] with $\mathrm{BCH}$ coding. While Saeedi et al. [18] examined the performance of hybrid method in Additive White Gaussian Noise (AWGN) channels, the performance of hybrid method was also studied in Rician channels by AliHemmati and Azmi [19]. In addition, another hybrid method was proposed in [20] by employing Reed-Solomon coding and least square method [17].

The downlink of Orthogonal Frequency-Division Multiple Access (OFDMA) system comprises many users with different modulations and channel codes who usually have no knowledge of modulation and channel code of the other users. Thus, methods such as [912] cannot be applied to OFDMA receivers because they must know in advance the modulation types and channel codes employed by the other users to reconstruct the clipped samples. On the other hand, the least square methods [17-19] are independent of modulation and channel code; thus, these methods can be used on OFDMAs when users have different modulations and channel codes with no knowledge about modulation and channel code of the other users.

There are a few subcarriers that carry zeros or certain symbols and they are known at the receiver side. Cen et al. [21] use those known subcarriers of OFDMA to estimate and to compensate the clipping noise by employing the least square equations whereas the transmitted signal has been clipped at Nyquist rate. The method in [21] uses a few known subcarriers (about $10 \%$ ) to reconstruct clipped samples successfully when the number of clipped samples is lower than that of known subcarriers. In comparison with [21], the methods [17-20] are more robust against AWGN and clipping noise, support lower clipping thresholds, and reconstruct more clipped samples because they use more equations in the least square stage. Nevertheless, in [17-20] need bandwidth expansion is needed to reconstruct clipped samples successfully while there is no need for more bandwidth in [21].

The objective of authors in this paper is to develop an improved version of [17] while making computations less complex. The proposed method converts the overdetermined system to a linear system by using constant pre-computed matrices and obtaining a pair of FFT/IFFT. The resulting linear system is then solved by using the Jacobi numerical method [22]. Finally, amplitudes of clipped samples are compensated by utilizing least square solution. To reconstruct amplitudes of clipped samples successfully, the proposed method needs bandwidth expansion similar to those reported in [17-20]. The proposed method offers low complexity and adequate performance, especially at low clipping ratios; thus, it can be exploited in most DFT-based least square methods, such as in [17-20]. The new approach can also be used to reconstruct clipped samples of OFDM systems and finds application with other structures including single-carrier and OFDMA.

The rest of the paper is organized as follows. The PAPR definition and clipping procedure are briefly discussed in Section 2. Section 3 considers reconstruction of clipped samples by employing the least square method and Section 4 describes the proposed method. Simulation and analysis of results are discussed in Section 5 and the paper ends with a conclusion in Section 6 .

\section{Preliminaries}

For an OFDM transmitter with $N$ subcarriers, the oversampled time-domain sequence $\{s(n)\}_{n=0}^{J N-1}$ is transmitted as: 


$$
s(n)=\frac{1}{\sqrt{J N}} \sum_{k=0}^{N-1} S(k) e^{\mathrm{j} 2 \pi n k / J N}
$$

where $\mathbf{j}=\sqrt{-1}, J$ is oversampling factor, $\{S(k)\}_{k=0}^{N-1}$ are data modulated symbols and $n=0,1, \cdots, J N-1$. The PAPR of signal $s(n)$ can be approximated as:

$$
\operatorname{PAPR}=\frac{\max _{n=0,1, \cdots, J N-1}|s(n)|^{2}}{E\left[|s(n)|^{2}\right]},
$$

where $|\cdot|$ and $E[\cdot]$ denote the amplitude and expectation operator. This approximation will be more precise when oversampling rate of 4 or greater is used [23].

To reduce the PAPR of OFDM signal, the clipping and filtering procedure can be used. The clipping procedure is a non-linear function, which restricts the amplitudes of samples and leaves phases unaffected. The clipped signal $\left\{s_{c}(n)\right\}_{n=0}^{J N-1}$ can be formulated as:

$$
s_{c}(n)= \begin{cases}s(n), & |s(n)| \leq T \\ T \frac{s(n)}{|s(n)|}, & |s(n)|>T\end{cases}
$$

where $T$ is clipping threshold and $n=0,1, \cdots, J N-1$.

The clipping procedure causes out-of-band radiations and these radiations should be removed by filtering procedure. The filtering procedure is done at frequency domain by zeroing out-of-band components:

$$
S_{C F}(k)= \begin{cases}S_{C}(k), & k=0,1, \cdots, N-1 \\ 0, & k=N, N+1, \cdots, J N-1\end{cases}
$$

where sequence $\left\{S_{C}(k)\right\}_{k=0}^{J N-1}$ is frequency domain of clipped sequence $\left\{S_{C}(n)\right\}_{n=0}^{J N-1}$. Finally, the timedomain sequence of $\left\{S_{C F}(k)\right\}_{k=0}^{J N-1}$ is transmitted after adding cyclic prefix to the sequence.

\section{Reconstruction of clipped signal}

Let $\{x(n), n=0,1, \cdots, J N-1\}$ be the received and equalized OFDM signal with oversampling rate of $J$ times. Let also sets of $\left\{l_{i}, i=1,2, \cdots, L\right\}$ and $\left\{p_{j}, j=\right.$ $1,2, \cdots, P\}$ be the time indices of clipped and nonclipped samples of $x(n)$, respectively, and $P=J N-$ $L$. The frequency-domain of sequence $\{x(n)\}_{n=0}^{J N-1}$ is computed by:

$$
X(k)=\frac{1}{\sqrt{J N}} \sum_{n=0}^{J N-1} x(n) e^{-\mathbf{j} 2 \pi n k / J N},
$$

where $k=0,1, \cdots, J N-1$.

Since the signal $\{x(n)\}_{n=0}^{J N-1}$ is oversampled $J$ times, the out-of-band components $\left\{X\left(k^{\prime}+N\right)\right.$ \}$_{k^{\prime}=0}^{(J-1) N-1}$ should be zero while in-band components

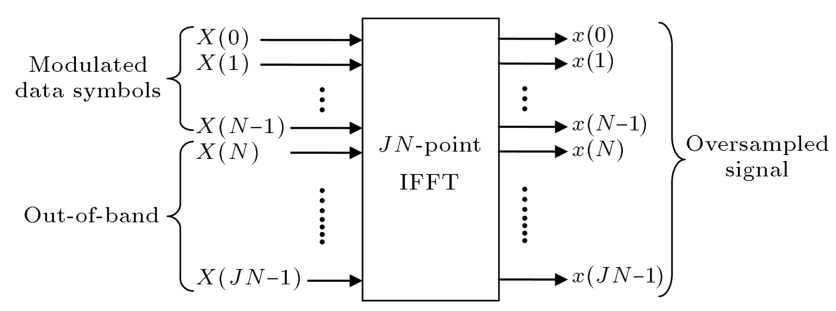

Figure 1. The frequency domain of oversampled signal $x(n)$ consists of $(J-1) N$ zeros and $N$ modulated data symbols.

$\left\{X\left(k^{\prime \prime}\right)\right\}_{k^{\prime \prime}=0}^{N-1}$ carry modulated data symbols, see Figure 1. By considering the out-of-band components, $(J-1) N$ equations can be expressed as:

$$
\begin{aligned}
& \frac{1}{\sqrt{J N}} \sum_{i=1}^{L} x\left(l_{i}\right) e^{-\mathrm{j} 2 \pi\left(k^{\prime}+N\right) l_{i} / J N} \\
& \quad=-\frac{1}{\sqrt{J N}} \sum_{j=1}^{P} x\left(p_{j}\right) e^{-\mathbf{j} 2 \pi\left(k^{\prime}+N\right) p_{j} / J N}
\end{aligned}
$$

where $x\left(l_{i}\right)$ and $x\left(p_{j}\right)$ are clipped and non-clipped samples, respectively, and $k^{\prime}=0,1, \cdots,(J-1) N-1$. If the clipped samples are assumed to be unknown, then, a linear system of $(J-1) N$ equations with $L$ unknowns is formulated. This system can be written in matrix form:

$$
\overline{\mathbf{W}} \overline{\mathbf{x}}=-\tilde{\mathbf{W}} \tilde{\mathbf{x}}
$$

where vectors $\overline{\mathbf{x}}$ and $\tilde{\mathbf{x}}$ comprise clipped and nonclipped samples of sequence $\{x(n), n=0,1, \cdots, J N-$ $1\}, \overline{\mathbf{W}}=\left[\bar{w}_{k^{\prime}, i}\right]_{(J-1) N \times L}$, respectively:

$$
\begin{aligned}
& \overline{\mathbf{x}}=\left[\begin{array}{llll}
x\left(l_{1}\right) & x\left(l_{2}\right) & \cdots & x\left(l_{L}\right)
\end{array}\right]^{T}, \\
& \tilde{\mathbf{x}}=\left[\begin{array}{llll}
x\left(p_{1}\right) & x\left(p_{2}\right) & \cdots & x\left(p_{P}\right)
\end{array}\right]^{T} .
\end{aligned}
$$

In additions, the two matrices $\overline{\mathbf{W}}=\left[\bar{w}_{k^{\prime}, i}\right]_{(J-1) N \times L}$ and $\tilde{\mathbf{W}}=\left[\tilde{w}_{k^{\prime}, j}\right]_{(J-1) N \times P}$ contain Fourier coefficients:

$$
\begin{aligned}
& \bar{w}_{k^{\prime}, i}=\frac{1}{\sqrt{J N}} e^{-\mathbf{j} 2 \pi\left(k^{\prime}+N\right) l_{i} / J N}, \\
& \tilde{w}_{k^{\prime}, j}=\frac{1}{\sqrt{J N}} e^{-\mathbf{j} 2 \pi\left(k^{\prime}+N\right) p_{j} / J N},
\end{aligned}
$$

where $i=1,2, \cdots, L, j=1,2, \cdots, P$ and $k^{\prime}=$ $0,1, \cdots,(J-1) N-1$.

In Eq. (7), the number of unknowns (clipped samples) is usually smaller than the number of equations. This equation has a unique least square solution because $\overline{\mathbf{W}}$ is a full rank matrix that has $L$ independent columns. The least square solution of $\mathrm{Eq} .(7)$ is obtained by solving the following linear system [22]:

$$
\overline{\mathbf{W}}^{H} \overline{\mathbf{W}} \overline{\mathbf{x}}=-\overline{\mathbf{W}}^{H} \tilde{\mathbf{W}} \tilde{\mathbf{x}}
$$


where $\overline{\mathbf{W}}^{H}$ denotes Hermitian (conjugate transpose) of $\overline{\mathbf{W}}$ [22]. This overdetermined linear system can be formulated as square linear system $\mathbf{A} \overline{\mathbf{x}}=\mathbf{b}$, where:

$$
\begin{aligned}
& \mathbf{A}=-\varepsilon \overline{\mathbf{W}}^{H} \overline{\mathbf{W}}, \\
& \mathbf{b}=\varepsilon \overline{\mathbf{W}}^{H} \tilde{\mathbf{W}} \tilde{\mathbf{x}}, \\
& \varepsilon=J /(J-1) .
\end{aligned}
$$

After solving linear system (Eq. (12)), the signal $x(n)$ can be compensated by replacing the clipped samples $\left\{x\left(l_{i}\right), i=1,2, \cdots, L\right\}$ with the solution $\overline{\mathbf{x}}$ of the linear system (Eq. (12)).

\section{Proposed method}

Preparation of the linear system $\mathbf{A} \overline{\mathbf{x}}=\mathbf{b}$ needs $(J-$ 1) $\left(L^{2}+L+P\right) N$ complex multiplications and direct solution of the linear system has complexity order of $O\left(L^{3}\right)$. In Subsection 4.1., a fast method to construct linear system at low complexity is described. This method only needs a pair of FFT/IFFT computations. In Subsection 4.2., $\mathbf{A} \overline{\mathbf{x}}=\mathbf{b}$ is solved numerically by invoking the Jacobi method. The amplitude reconstruction of clipped samples is explained in Subsection 4.3. and the transmitter and receiver parts of the proposed method are described in the last subsection.

\subsection{Preparing linear system}

This subsection and the next describe the way authors prepare and solve linear system (Eq. (12)) by using a pre-computation step, an FFT/IFFT pair, and the Jacobi numerical method.

Vector $\mathbf{b}$ can be computed by implementing FFT and IFFT in two steps. In the first step, $\mathbf{c}=\tilde{\mathbf{W}} \tilde{\mathbf{x}}$ is computed by employing FFT and the second step uses IFFT to evaluate $\mathbf{b}=\varepsilon \overline{\mathbf{W}}^{H} \mathbf{c}$.

For the first step, vector $\mathbf{y}=\left[y_{n}\right]_{J N \times 1}$ and incomplete Fourier matrix $\mathbf{W}=\left[w_{k^{\prime}, n}\right]_{(J-1) N \times J N}$ are defined as:

$$
\begin{aligned}
& y_{n}= \begin{cases}x(n), & n \in\left\{l_{i}, i=1,2, \cdots, L\right\} \\
0, & n \in\left\{p_{j}, j=1,2, \cdots, P\right\}\end{cases} \\
& w_{k^{\prime}, n}=\frac{1}{\sqrt{J N}} e^{-\mathbf{j} 2 \pi\left(k^{\prime}+N\right) n / J N},
\end{aligned}
$$

where $k^{\prime}=0,1, \cdots,(J-1) N-1$ and $n=$ $0,1, \cdots, J N-1$. Based on these definitions, the products $\tilde{\mathbf{W}} \tilde{\mathbf{x}}$ and $\mathbf{W y}$ are equal. Since the matrix $\mathbf{W}$ contains bottom rows of Fourier matrix, $\mathbf{W y}$ is equal to the last $(J-1) N$ component of $\operatorname{FFT}(\mathbf{y})$ :

$$
\begin{aligned}
& \mathbf{z}=\operatorname{FFT}(\mathbf{y}), \\
& \mathbf{c}=\left[\begin{array}{llll}
z_{N} & z_{N+1} & \cdots & z_{J N-1}
\end{array}\right]^{T} .
\end{aligned}
$$

In the second step, vector $\mathbf{t}=\left[t_{i}\right]_{J N \times 1}$ is constructed by using the last components of $\mathbf{z}$ :

$$
t_{i}= \begin{cases}z_{k}, & N \leq k \leq J N-1 \\ 0, & 0 \leq k \leq N-1\end{cases}
$$

The above equation implies that product $\mathbf{b}=\varepsilon \overline{\mathbf{W}}^{H} \mathbf{c}$ is same as $\varepsilon \mathbf{W}^{H} \mathbf{t}$. These products can be computed by using IFFT and selecting certain components of IFFT outputs:

$$
\begin{aligned}
\mathbf{u} & =\operatorname{IFFT}(\mathbf{t}), \\
\mathbf{b} & =\varepsilon\left[\begin{array}{llll}
u_{l_{1}} & u_{l_{2}} & \cdots & u_{l_{L}}
\end{array}\right]^{T} .
\end{aligned}
$$

After preparing vector $\mathbf{b}$, we must construct matrix A. For this aim, strictly diagonally dominant matrix $\mathbf{C}=\left[c_{n, n^{\prime}}\right]_{J N \times J N}$ is defined as:

$$
\mathbf{C}=-\mathbf{W}^{H} \mathbf{W} \text {. }
$$

Matrix $\mathbf{C}$ is a constant matrix for all OFDM symbols and needs to be computed once. On the other hand, matrix $\mathbf{A}$ can be constructed for each OFDM symbol by selecting certain components of matrix $\mathbf{C}$ :

$$
a_{i, i^{\prime}}=c_{l_{i}, l_{i^{\prime}}}, \quad i, i^{\prime} \in\{1,2, \cdots, L\} .
$$

Matrix $\mathbf{C}$ is independent of $\left\{l_{i}\right\}_{i=1}^{L}$ and can be computed once. This matrix is a $J N \times J N$ Hermitian matrix with $-\mathbf{1} \mathrm{s}$ on its diagonal. Therefore, the algorithm needs $J N(J N-1)$ store locations.

By accessing a few of these store locations, matrix A can be constructed as:

$$
a_{i, i^{\prime}}=-\frac{1}{(J-1) N} \sum_{k^{\prime}=0}^{(J-1) N-1} e^{\mathrm{j} 2 \pi\left(l_{i}-l_{i^{\prime}}\right)\left(k^{\prime}+N\right) / J N}
$$

Based on geometric series properties, Eq. (25) can be simplified as:

$$
a_{i, i^{\prime}}=a_{i^{\prime}, i}^{*}= \begin{cases}\frac{\theta\left(l_{i}, l_{i^{\prime}}\right)}{1-\varphi\left(l_{i}\right) \varphi^{*}\left(l_{i^{\prime}}\right)}, & i \neq i^{\prime} \\ -1, & i=i^{\prime}\end{cases}
$$

where $a_{i^{\prime}, i}^{*}$ is conjugate of $a_{i^{\prime}, i}$, and $\theta\left(l_{i}, l_{i^{\prime}}\right)$ and $\varphi_{i}$ are defined as:

$$
\begin{aligned}
& \theta\left(l_{i}, l_{i^{\prime}}\right)=\frac{1-e^{\mathrm{j} 2 \pi\left(l_{i}-l_{i^{\prime}}\right) / J}}{(J-1) N}, \\
& \varphi\left(l_{i}\right)=e^{\mathrm{j} 2 \pi l_{i} / J N} .
\end{aligned}
$$

In this paper, we choose $J=4$; thus, Eq. (27) is simplified as: 


$$
\theta\left(l_{i}, l_{i^{\prime}}\right)= \begin{cases}0, & \bmod \left(l_{i}-l_{i^{\prime}}, 4\right)=0 \\ \frac{1-\mathbf{j}}{3 N}, & \bmod \left(l_{i}-l_{i^{\prime}}, 4\right)=1 \\ \frac{2}{3 N}, & \bmod \left(l_{i}-l_{i^{\prime}}, 4\right)=2 \\ \frac{1+\mathbf{j}}{3 N}, & \bmod \left(l_{i}-l_{i^{\prime}}, 4\right)=3\end{cases}
$$

where mod indicates the modulus after division. In this case, the terms $\theta\left(l_{i}, l_{i^{\prime}}\right)$ and $\varphi\left(l_{i}\right)$ have only 4 and $4 N$ variations, respectively. All variations of $\theta\left(l_{i}, l_{i^{\prime}}\right)$ and $\varphi\left(l_{i}\right)$ can be stored in memory as pre-computed values in order to avoid increasing complexity. On the other hand, computation of each $a_{i, i^{\prime}}, i \neq i^{\prime}$ needs a complex multiplication, a complex division, and a real addition based on Eq. (27). Therefore, construction of A needs $L(L-1)$ complex multiplications and divisions and $L(L-1) / 2$ additions.

After preparation, the linear system $\mathbf{A} \overline{\mathbf{x}}=\mathbf{b}$ will be solved by utilizing the Jacobi numerical method whose complexity order of each iteration is $O\left(L^{2}\right)$.

\subsection{Solving linear system}

The Jacobi method begins with an initial approximation $\overline{\mathbf{x}}^{(0)}$ of the solution vector. Then, a sequence of approximations $\left\{\overline{\mathbf{x}}^{(t)}\right\}$ is generated iteratively:

$$
\overline{\mathbf{x}}^{(t+1)}=\mathbf{D} \overline{\mathbf{x}}^{(t)}+\mathbf{e}, \quad t=1,2, \cdots, t_{\max },
$$

where $t$ denotes iteration number, $\mathbf{D}=\left[d_{i, i^{\prime}}\right]_{L \times L}$ and $\mathbf{e}=\left[e_{i}\right]_{L \times 1}$ are Jacobi iteration matrix and Jacobi vector, respectively. Since matrix $\mathbf{A}$ has $-\mathbf{1}$ s along the main diagonal, Jacobi vector is defined as $\mathbf{e}=-\mathbf{b}$ and components of Jacobi matrix $\mathbf{D}$ are expressed as:

$$
d_{i, i^{\prime}}= \begin{cases}0, & i=i^{\prime} \\ a_{i, i^{\prime}} & i \neq i^{\prime}\end{cases}
$$

for $i, i^{\prime} \in\{1,2, \cdots, L\}$.

Based on Eq. (13), A is strictly diagonally dominant matrix [24]. The strictly diagonally dominant property makes sequence Eq. (30) converge on the solution of linear system [22].

If the clipped samples are used as initial approximation $\overline{\mathbf{x}}^{(0)}$, then Eq. (17) converges on the solution after 5 to 10 iterations. The Jacobi method needs $L(L-1)$ complex multiplications and $L(L-1)$ complex additions for each iteration.

\subsection{Amplitude reconstruction}

The clipping procedure only reduces amplitude of samples that are large and leaves phases of all samples unchanged. After clipping, the clipped samples still have large amplitudes and, thus, their phases have less impressibility against AWGN than those with lower amplitudes. This property is illustrated in Figure 2. Based on this property, only the amplitudes of clipped

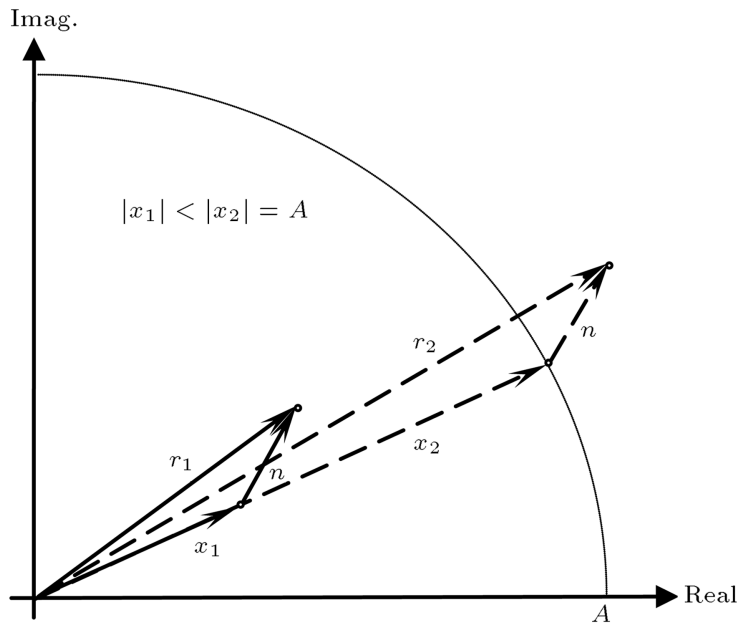

Figure 2. The additive noise, $n$, has less effect on the phases of samples with greater amplitudes.

samples should be reconstructed at the receiver and it is not necessary to reconstruct the phases [10-11]. While Saeedi et al. [17] uses the complex value of linear system solution to replace clipped samples, this paper uses its amplitude to replace amplitude of clipped samples. This technique improves BER performance compared to $[17]$.

\subsection{Transmitter and receiver}

Figure 3 shows the block diagrams of the transmitter and receiver for the proposed method. As can be seen, the receiver includes filtering and equalizing processes before amplitude reconstruction stage. The receiver uses a zero-force equalizer [25] to reduce the effects of channel. On the other hand, the filtering process removes the parts of frequency domain that only contain AWGN. The use of filtering and equalizing processes, therefore, helps the linear system to endure less noise and perturbation.

In order to construct the linear system represented by $\mathbf{A} \overline{\mathbf{x}}=\mathbf{b}$, the location of clipped samples must be known. This calls for examination of two points; firstly, we know Eq. (6) is valid for $k^{\prime}=$ $0,1, \cdots,(J-1) N-1$; therefore, up to $(J-1) N$ unknown samples can be found by using Eq. (6) and the succeeding equations. If $L^{\prime}$ non-clipped samples (in addition to $L$ clipped samples) are taken as unknowns, then $L+L^{\prime}$ unknowns can be obtained by Eq. (6) where $L+L^{\prime}$ is smaller than $(J-1) N$. Secondly, by considering the effect of the AWGN, we know that the amplitudes of samples have changed in a way that most of the equalized clipped samples have amplitudes within intervals $\left[T-\eta \sigma_{N}, T+\eta \sigma_{N}\right]$, where $\sigma_{N}$ is the variance of AWGN, $T$ is the clipping threshold, and $\eta \geq$ 0 . Chen et al. [21] use the threshold $T-\eta \sigma_{N}$ to estimate the locations of clipped samples. In this manner, the clipped samples can be reconstructed approximately by comparing the amplitude of equalized signal with the 


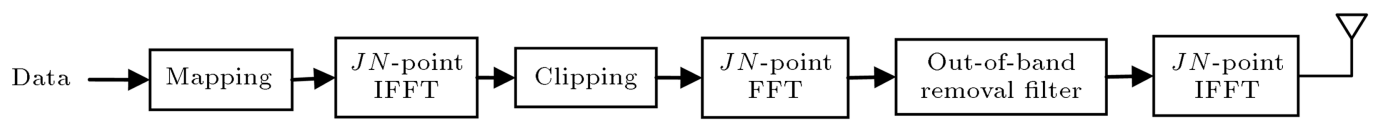

(a)



(b)

Figure 3. Block diagrams of the proposed method: (a) Transmitter and (b) receiver.

threshold $T-\eta \sigma_{N}$ [21]. If the amplitude of the sample is greater than the threshold, the sample is probably a clipped sample. Hence, this technique finds most of the clipped samples, although some of non-clipped samples are also extracted as clipped samples, which have no effect on the reconstruction process itself as there are $(J-1) N$ equations outnumbering the unknowns.

After solving linear system, $\mathbf{A} \overline{\mathbf{x}}=\mathbf{b}$, the amplitudes of clipped samples are reconstructed by replacing them with those obtained from the solution of linear system and the phases of clipped samples are left unchanged.

\subsection{Comparison with other methods}

The iterative techniques [9-12] exploit modulation and channel coding information to estimate and reconstruct the clipped signal. These methods reconstruct the clipped samples by employing demodulation/modulation with channel decoding/coding processes iteratively. Therefore, the receiver has to know the modulation and channel coding types of all users when iterative methods [9-12] are employed for OFDMA systems.

On the other hand, techniques of [17-20] are founded on DFT equations. These methods approximate the clipped samples by preparing a linear system and solve the equations to specify the complex values of clipped samples. These methods have two important disadvantages: high complexity and instability. They require high computational complexity to prepare and solve a non-square linear system and cannot reconstruct the clipped samples, especially when the perturbation caused by the clipping and filtering procedure is high.

In contrast to [17-20], the proposed method prepares DFT equations simply and solves the linear system iteratively. The proposed method improves performance of DFT-based methods by employing the Jacobi numerical scheme and amplitude reconstruction technique while the overall complexity of the proposed method is efficiently decreased. The complexity of our method is reduced by preparing a square linear system $\mathbf{A} \overline{\mathbf{x}}=\mathbf{b}$ instead of the overdetermined DFT-based system (Eqs. (7)). The matrix $\mathbf{A}$ is prepared simply by utilizing (25)-(29) and the vector $\mathbf{b}$ is computed by obtaining a deficient FFT/IFFT pair. Several numerical schemes can be exploited to solve the square linear system, $\mathbf{A} \overline{\mathbf{x}}=\mathbf{b}$. Among these methods, we choose Jacobi scheme to reduce complexity and increase stability of the solution against the perturbations, which are imposed by AWGN, clipping, and filtering procedures.

\section{Simulation and analysis}

At this stage, simulation of OFDM system is performed by selecting $N=1024$ subcarriers, 16-QAM constellation, and a $5-\mathrm{MHz}$ bandwidth. For the proposed method, $J=4$ times oversampled signal is used for the clipping and filtering stage because $J=4$ is sufficient for PAPR reduction purposes. The results of simulation and analysis are presented in the following subsections.

\subsection{Complementary cumulative distribution function}

Complementary Cumulative Distribution Functions (CCDFs) of the PAPR for common OFDM and several cases of clipped OFDM are plotted using an oversampling factor of 16 in Figure 4. During clipping procedure, OFDM signals are clipped at various Clipping

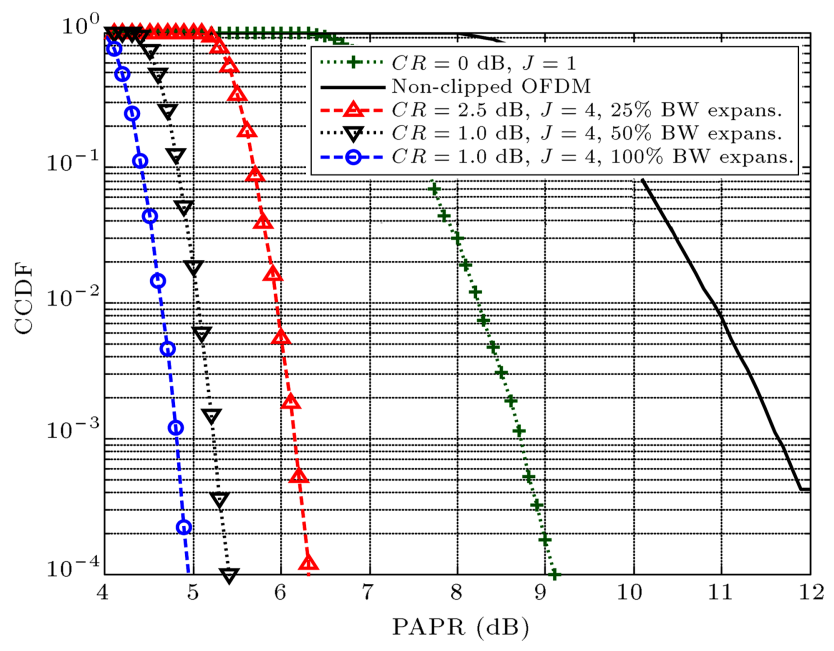

Figure 4. PAPR CCDFs of OFDM in several situations. As shown in the figure, bandwidth expansion reduces PAPR. 
Ratios (CRs), which are estimated by:

$$
C R=10 \log _{10} \frac{T^{2}}{E\left[|s(n)|^{2}\right]},
$$

where $T$ is the clipping threshold. For the case of $C R=$ $0 \mathrm{~dB}$ and $J=1$, clipping is employed at Nyquist rate and for the remaining clipping cases, signals are clipped at oversampling rate of $J=4$.

Figure 4 verifies that the clipping and filtering methods can achieve low PAPR compared to clipping at Nyquist rate and that bandwidth expansion also reduces this parameter. Based on this figure, PAPR of the proposed method can be even lower than clipping at Nyquist rate.

In the following subsection, we will evaluate our BER performance when the bandwidth is expanded by $25 \%$ and $100 \%$ at clipping ratios of $2.5 \mathrm{~dB}$ and $1.0 \mathrm{~dB}$, respectively.

\subsection{BER performance}

In order to assess BER performance of the proposed method, Rayleigh and Rician channels are used for simulations. The channel frequency response of the $m$ th OFDM symbols at $k$ th subcarrier is denoted by:

$$
H_{m}(k)=\sum_{d=1}^{D} \alpha_{d, m} e^{-\mathbf{j} 2 \pi k \Delta f \tau_{d}}
$$

where $\alpha_{d, m}$ is the complex amplitude and $\tau_{d}$ is the delay of the $d$ th path and $\Delta f$ is the subcarrier separation in the frequency domain. $\alpha_{2, m}, \alpha_{3, m}, \cdots$ and $\alpha_{D, m}$ are modeled as zero mean complex Gaussian random variables with variances $\sigma_{2}^{2}, \sigma_{3}^{2}, \cdots$ and $\sigma_{D}^{2}$. $\alpha_{1, m}$ is modeled as a complex Gaussian random variable with variance $\sigma_{1}^{2}$ while its mean is zero for Typical Urban (TU) channel and $\sqrt{K \sigma_{1}^{2}}$ for Typical Rural Area (TRA) channel with Rician $K$-factor. In this paper, the Rician $K$-factor is set to 1.0. The delays $\tau_{d}$ and variances $\sigma_{d}^{2}$ of channels are displayed in Table 1 based on COST207 channel models [26].

In this subsection, the BER performances versus Signal-to-Noise Ratio (SNR) are plotted. The SNR is defined as:

$$
\mathrm{SNR}=\frac{P_{S} P_{H}}{P_{Z}},
$$

where $P_{Z}, P_{S}$, and $P_{H}$ are average power of AWGN, transmitted signal, and channel fade $H_{m}$, respectively.

The BER performances over Rayleigh and Rician channels are plotted in Figures 5 and 6 , when the receiver knows the locations of the clipped samples. The analytical BER of conventional OFDM is plotted based on [25]. As can be seen, the proposed method suppresses the clipping noise effectively while [17] cannot reconstruct the clipped samples at low clipping
Table 1. The delays and powers of channel taps.

\begin{tabular}{ccc}
\hline \multicolumn{3}{c}{ (a) Typical Rural Area (TRA) } \\
\hline Tap number & Delay $(\boldsymbol{\mu s})$ & Power $(\mathbf{d B})$ \\
\hline 1 & 0.0 & 0.602 \\
2 & 0.1 & 0.241 \\
3 & 0.2 & 0.096 \\
4 & 0.3 & 0.036 \\
5 & 0.4 & 0.018 \\
6 & 0.5 & 0.006 \\
\hline
\end{tabular}

(b) Typical Urban (TU)

\begin{tabular}{ccc} 
Tap number & Delay $(\boldsymbol{\mu} \mathbf{s})$ & Power $(\mathbf{d B})$ \\
\hline 1 & 0.0 & -3 \\
2 & 0.2 & 0 \\
3 & 0.5 & -2 \\
4 & 1.6 & -6 \\
5 & 2.3 & -8 \\
6 & 5.0 & -10 \\
\hline
\end{tabular}

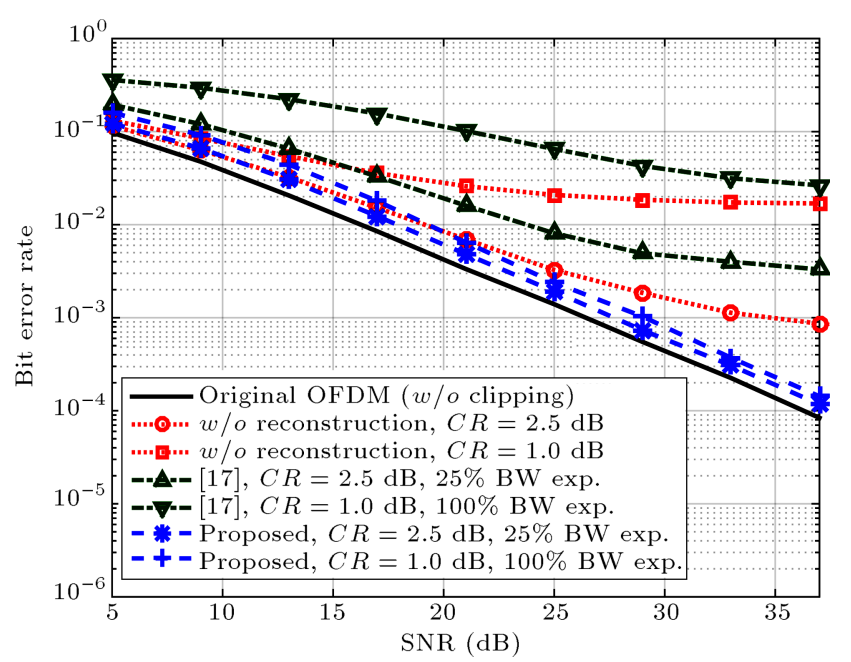

Figure 5. BER performance over TU channel model when receiver knows the locations of clipped samples. As shown, the proposed method has low BER degradations with respect to non-clipped (original) OFDM signal.

ratios such as $1 \mathrm{~dB}$ and $2.5 \mathrm{~dB}$. Hence, the proposed method has better stability than [17] at lower clipping ratios because it only reconstructs amplitudes by using iterative Jacobi sequence (Eq. (30)). Figure 7 illustrates effects of Jacobi numerical scheme and amplitude reconstruction method on error performances. This figure implies that both of the mentioned techniques increase stability of reconstruction.

Figure 8 depicts the BER performances of the proposed method over TRA channel when the receiver does not know the locations of the clipped samples and must estimate them. The locations of clipping are estimated approximately by using a simple thresh- 


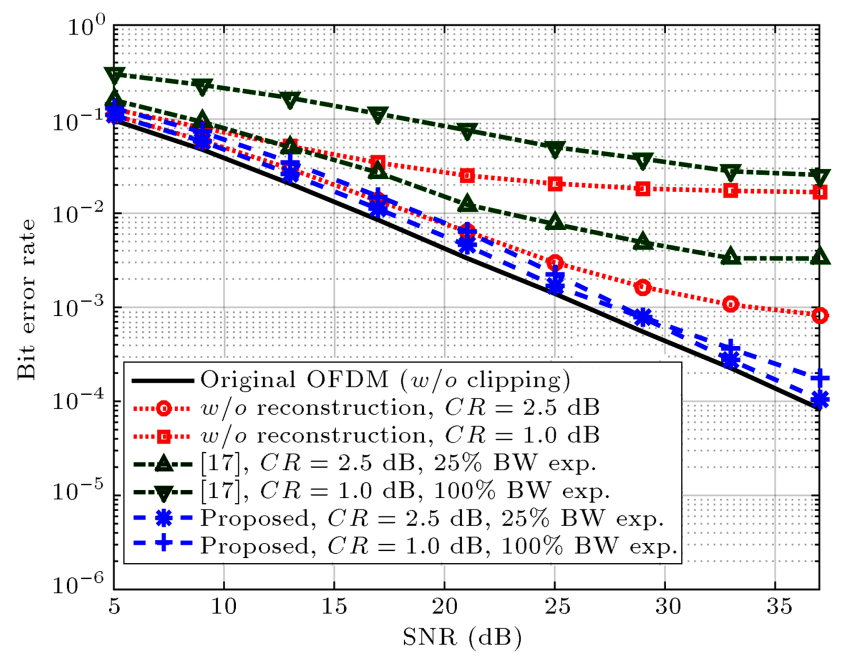

Figure 6. BER performance over TRA channel when receiver knows the locations of clipped samples. As shown, the proposed method has low BER degradations with respect to non-clipped (original) OFDM signal.

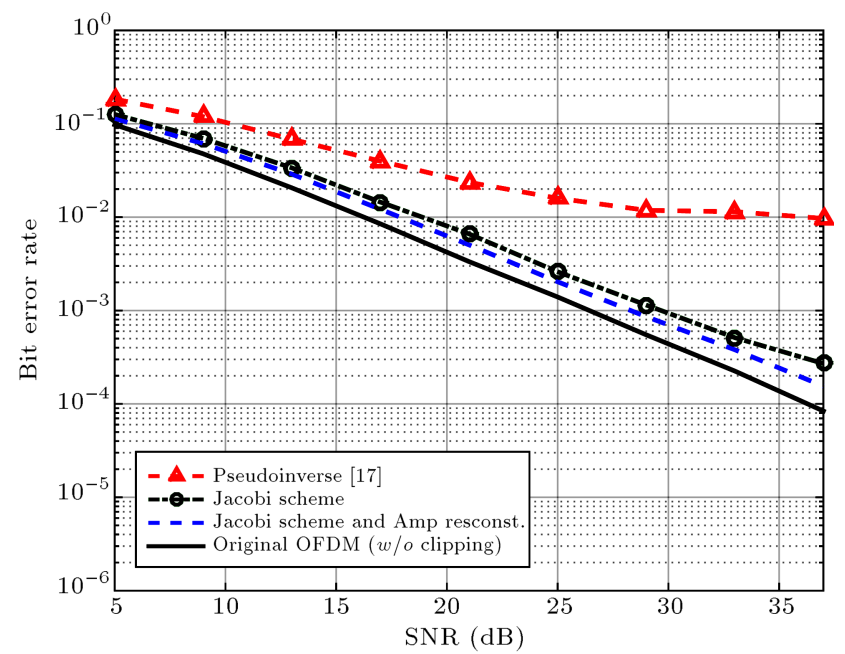

Figure 7. Effects of Jacobi numerical scheme and amplitude reconstruction technique on error performances when $C R=2(\mathrm{~dB})$.

old. Then, linear system, $\mathbf{A} \overline{\mathbf{x}}=\mathbf{b}$, is prepared by employing the estimated locations and reconstructing their amplitudes based on the solution of linear system. As Figure 8 illustrates, performance of the proposed method can be acceptable even when receiver has no knowledge of locations of clipped samples.

\subsection{Complexity analysis}

Our proposed method uses oversampled signal clipping and filtering at the transmitter and, at the other side, the receiver compensates BER degradation by reconstructing amplitude of clipped samples. The proposed reconstruction method requires a pair of FFT/IFFT and $L^{2}$ multiplications in order to prepare the Jacobi matrix and Jacobi vector. The sequence (30) also needs $L(L-1)$ multiplications in each iteration.

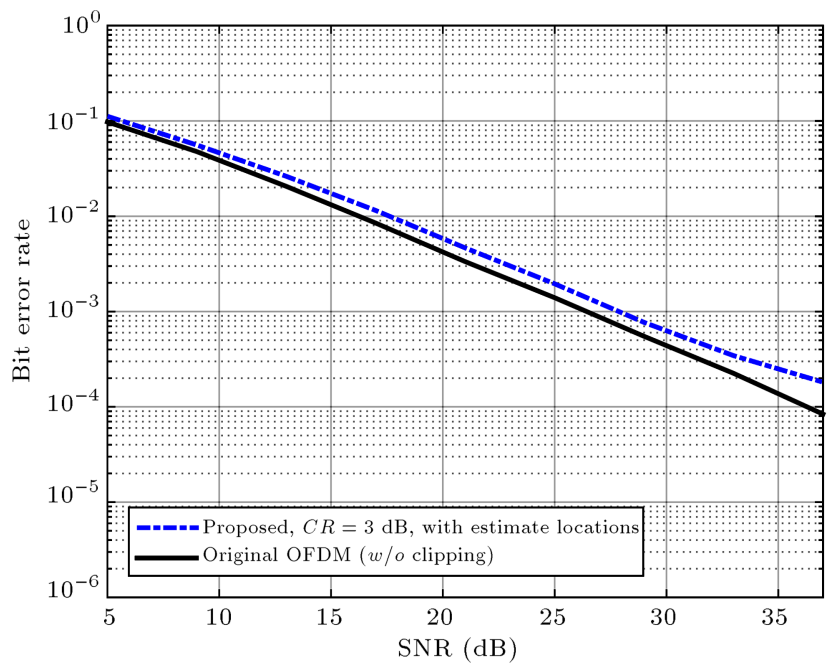

Figure 8. BER performance over TRA channel when receiver does not know the locations of clipped samples.

Thus, the overall complexity of the proposed method is $L(L-1) t_{\max }+L^{2}+J N \log _{2}(J N)$ complex multiplications/divisions and $L(L-1) t_{\max }+2 J N \log _{2}(J N)$ complex additions at the receiver where $t_{\max }$ indicates the maximum number of iterations.

On the other hand, we know that System (7) can be written as Vandermonde matrices and solved by the Vandermonde least square methods as in [2729]. The order of complexities of these methods is $O\left((J-1) N L+L^{2}\right)$, whereas the complexity order of the proposed technique is $O\left(L^{2}\right)$. For a reasonable PAPR value, the number of clipped samples is usually much less than the total number of out-of-band components, i.e. $L<<(J-1) N$. Therefore, the complexity order of the proposed solution is less than those of [27-29].

For the aforementioned OFDM system, the approximate numbers of multiplications and divisions carried out in the proposed and other methods are listed in Table 2. Based on this table, the proposed method reduces the complexity of [17] more than 300 times.

Table 2. Approximate comparison of complexities.

\begin{tabular}{lc}
\hline Method & $\begin{array}{c}\text { Approximate number of } \\
\text { multiplications /divisions }\end{array}$ \\
\hline$[17], C R=1.0 \mathrm{~dB}$ & $4.3 \times 10^{9}$ \\
{$[17], C R=2.5 \mathrm{~dB}$} & $1.6 \times 10^{9}$ \\
Proposed, & \\
$C R=1.0 \mathrm{~dB}$, & $1.2 \times 10^{7}$ \\
10 iterations & \\
Proposed, & \\
$C R=2.5 \mathrm{~dB}$, & $4.8 \times 10^{6}$ \\
10 iterations & \\
\hline
\end{tabular}




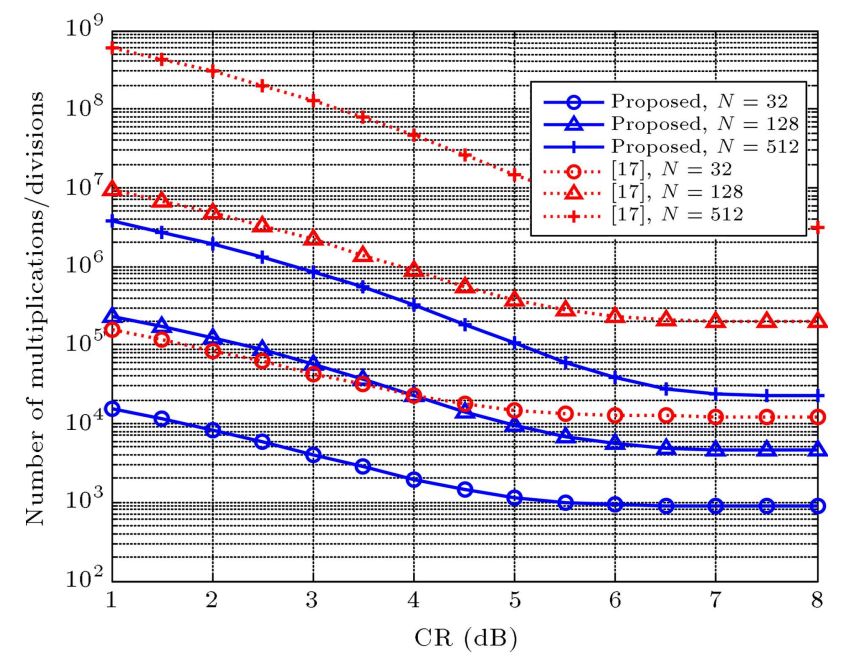

Figure 9. Average number of multiplications/divisions required for [17] and the proposed method for OFDM systems with 16-QAM and $N$ subcarriers.

The average numbers of multiplications/divisions for various clipping ratios and several cases of $N$ are plotted in Figure 9. This figure shows that complexity of the proposed method is much less than that of the other method.

\section{Conclusion}

This paper introduced an innovative amplitude reconstruction method and demonstrated that it was capable of offering superior features for clipped signals (OFDM, OFDMA, or single-carrier). The proposed model used clipping and filtering technique at the transmitter to reduce the PAPR and reconstruct the clipped samples at the receiver without any knowledge about modulation and channel code. It was found that bandwidth expansion was required in order to reconstruct the clipped samples effectively at a reasonable degree of complexity. The presented algorithm has the potential to be used in other similar approaches such as [17-21] where DFT-based least square equations are required. Simulation results confirm its outstanding BER performance at reasonable PAPR compared to the similar methods considered.

\section{References}

1. Han, S.H. and Lee, J.H. "An overview of peak-toaverage power ratio reduction techniques for multicarrier transmission", IEEE Wireless Communications, 12(2), pp. 56-65 (2005).

2. Armstrong, J. "Peak-to-average power reduction for OFDM by repeated clipping and frequency domain filtering", IEE Electronics Letters, 38, pp. 246-247 (2002).

3. Han, S.H. and Lee, J.H. "PAPR reduction of OFDM signals using a reduced complexity PTS technique",
IEEE Signal Processing Letters, 11, pp. $887-890$ (2004).

4. Youa, Y.H., Jungb, H.K. and Jeonc, W.G. "A simple peak-to-average power ratio (PAR) reduction scheme for OFDM-CDMA signals", International Journal of Electronics and Communications, 58, pp. 142-146 (2004).

5. Breiling, H., Müller-Weinfurtner, S.H. and Huber, J.B. "SLM peak-power reduction without explicit side information", IEEE Communications Letters, 5, pp. 239-241 (2001).

6. Naeiny, M.F. and Marvasti, F. "PAPR reduction of space-frequency coded OFDM systems using active constellation extension", International Journal of Electronics and Communications, 65, pp. 873-878 (2011).

7. Vallavaraja, A., Stewartb, B.G. and Harrisonb, D.K. "An evaluation of modified $\mu$-law companding to reduce the PAPR of OFDM systems", International Journal of Electronics and Communications, 64, pp. 844-857 (2010).

8. Wang, Y. and Luo, Z. "Optimized iterative clipping and filtering for PAPR reduction of OFDM signals", IEEE Trans. Communications, 59, pp. 33-37 (2011).

9. Kim, D. and Stüber, G.L. "Clipping noise mitigation for OFDM by decision-aided reconstruction", IEEE Communications Letters, 3, pp. 4-6 (1999).

10. Kwon, U.K. and Im, G.H. "Iterative amplitude reconstruction of clipped OFDM signals with optimum equalization", IEE Electronics Letters, 42, pp. 11891190 (2006).

11. Kwon, U.K., Kim, D. and Im, G.H. "Amplitude clipping and iterative reconstruction of MIMO-OFDM signals with optimum equalization", IEEE Trans Wireless Communications, 8(1), pp. 268-277 (2009).

12. Chen, H. and Haimovish, M. "Iterative estimation and cancellation of clipping noise for OFDM signals", IEEE Communications Letters, 7, pp. 305-307 (2003).

13. Momenai, A. and Talebi, S. "Improving the stability of the DFT error recovery codes by using the Vandermonde fast decoding algorithm", IEEE International Conference on Acoustics, Speech, and Signal Processing, pp. 856-859 (2006).

14. Marvasti, F., Hasan, M., Echart, M. and Talebi, S. "Efficient techniques for burst error recovery", IEEE Trans Signal Processing, 47, pp. 1065-1075 (1999).

15. Marvasti, F., Nonuniform Sampling: Theory and Practice, New York, USA: Kluwer Academic/Plenum (2001).

16. Marvasti, F. "Spectrum of nonuniform samples", IEE Electronics Letters, pp. 896-897 (1984).

17. Saeedi, H., Sharif, M. and Marvasti, F. "Clipping noise cancellation in OFDM systems using oversampled signal reconstruction", IEEE Communications Letters, $\mathbf{6}$, pp. $73-75$ (2002). 
18. Saeedi, H., Azmi, P. and Marvasti, F. "A novel DFT-based method for clipping noise suppression in OFDM systems", IEEE Wireless Communications and Networking, New Orleans, USA, pp. 26-31 (2003).

19. AliHemmati, R. and Azmi, P. "Clipping distortion mitigation in OFDM systems over fading channels by using DFT-based method", Computers and Electrical Engineering, 31, pp. 431-443 (2005).

20. Tang, J., Jin, X., Zhang, X. and Cai, W. "A hybrid technique based on RS coding and least square method for clipping distortion mitigation in OFDM System", IEEE International Conference on Wireless Communications, Networking and Mobile Computing, Wuhan, China, pp. 1-4 (2006).

21. Chen, Y., Zhang, J. and Jayalath, A.D.S. "Estimation and compensation of clipping noise in OFDMA systems", IEEE Trans Wireless Communications, 9, pp. 523-527 (2010).

22. Datta, B.N., Numerical Linear Algebra and Applications, Pacific Grove, CA, USA: Brooks/Cole Publishing Company (1995).

23. Ochiai, H. and Imai, H. "On clipping for peak power reduction of OFDM signals", IEEE Vehicular Technology Conf., Tokyo, Japan, 2, pp. 731-735 (2000).

24. Gentle, J.E., Matrix Algebra: Theory, Computations, and Applications in Statistics, Springer (2007).

25. Cho, Y.S., Kim, J., Yang, W.Y. and Kang, C.G., MIMO-OFDM Wireless Communications with MAT$L A B$, John Wiley \& Sons (Asia) (2010).

26. COST 207. Management Committee. COST 207: Digital Land Mobile Radio Communications: Final Report (1989).
27. Demeure, C.J. "Fast QR factorization of Vandermonde matrices", Linear Algebra Appl., 122, pp. 165-194 (1989).

28. Demeure, C.J. and Thomson-CSF, J. "QR factorization of confluent Vandermonde matrices", IEEE Trans Acoustics, Speech and Signal Processing, 38, pp. 17991802 (1990).

29. Reichel, L. "Fast QR decomposition of Vandermondelike matrices and polynomial least squares approximation", SIAM J. Matrix Anal. Appl., 12, pp. 552-564 (1991).

\section{Biographies}

Adel Ahmadi received the BS, MS, and PhD degrees in Electrical Engineering from Shahid Bahonar University of Kerman, Iran, in 2008, 2010, and 2015, respectively. His research interests include image and video processing, space-time-frequency coding, OFDM systems, and distributed space-time-frequency coding in cooperative communication systems.

Siamak Talebi received BS and MS degrees in Communication Engineering from Isfahan University of Technology, in 1989 and 1992, respectively, and a PhD degree from the University of London (King's College), in 2001. He is currently with the Department of Electrical Engineering at Shahid Bahonar University of Kerman, Iran, and the Advanced Communications Research Institute at Sharif University of Technology, Tehran, Iran. His research interests include wireless communications, cognitive radio, MIMO-OFDM, and video coding. 